\title{
Sum Secrecy Rate in MISO Full-Duplex Wiretap Channel with
}

\section{Imperfect CSI}

\author{
Sanjay Vishwakarma and A. Chockalingam \\ sanjay@ece.iisc.ernet.in, achockal@ece.iisc.ernet.in \\ Department of ECE, Indian Institute of Science, Bangalore 560012
}

\begin{abstract}
In this paper, we consider the achievable sum secrecy rate in MISO (multiple-input-single-output) full-duplex wiretap channel in the presence of a passive eavesdropper and imperfect channel state information (CSI). We assume that the users participating in full-duplex communication have multiple transmit antennas, and that the users and the eavesdropper have single receive antenna each. The users have individual transmit power constraints. They also transmit jamming signals to improve the secrecy rates. We obtain the achievable perfect secrecy rate region by maximizing the worst case sum secrecy rate. We also obtain the corresponding transmit covariance matrices associated with the message signals and the jamming signals. Numerical results that show the impact of imperfect CSI on the achievable secrecy rate region are presented.
\end{abstract}

keywords: MISO, full-duplex, physical layer security, secrecy rate, semidefinite programming.

\section{INTRODUCTION}

Transmitting messages with perfect secrecy using physical layer techniques was first studied in [1] on a physically degraded discrete memoryless wiretap channel model. Later, this work was extended to more general broadcast channel in [2] and Gaussian channel in [3], respectively. Wireless transmissions, being broadcast in nature, can be easily eavesdropped and hence require special attention to design modern secure wireless networks. Secrecy rate and capacity of pointto-point multi-antenna wiretap channels have been reported in the literature by several authors, e.g., [4]-[7]. In the above works, the transceiver operates in half-duplex mode, i.e., either it transmits or receives at any given time instant. On the other hand, full-duplex operation gives the advantage of simultaneous transmission and reception of messages [8]. But loopback self-interference and imperfect channel state information (CSI) are limitations. Full-duplex communication without secrecy constraint has been investigated by many authors, e.g., [9]-[12]. Full-duplex communication with secrecy constraint has been investigated in [13]-[15], where the achievable secrecy rate region of two-way (i.e., fullduplex) Gaussian and discrete memoryless wiretap channels have been characterized. In the above works, CSI in all the links are assumed to be perfect.

In this paper, we consider the achievable sum secrecy rate in MISO full-duplex wiretap channel in the presence of a passive eavesdropper and imperfect CSI. The users participating in full-duplex communication have multiple transmit antennas, and single receive antenna each. The eavesdropper is assumed to have single receive antenna. The norm of the CSI errors in all the links are assumed to be bounded in their respective absolute values. In addition to a message signal, each user transmits a jamming signal in order to improve the secrecy rates. The users operate under individual power constraints. For this scenario, we obtain the achievable perfect secrecy rate region by maximizing the worst case sum secrecy rate. We also obtain the corresponding transmit covariance matrices associated with the message signals and the jamming signals. Numerical results that illustrate the impact of imperfect CSI on the achievable secrecy rate region are presented. We also minimize the total transmit power (sum of the transmit powers of users 1 and 2) with imperfect CSI subject to receive signal-to-interference-plusnoise ratio (SINR) constraints at the users and eavesdropper, and individual transmit power constraints of the users.

The rest of the paper is organized as follows. The system model is given in Sec. III Secrecy rate for perfect CSI is presented in Sec. III. Secrecy rate with imperfect CSI is studied in Sec. IV Results and discussions are presented in Sec. V. Conclusions are presented in Sec. VI

Notations : $\boldsymbol{A} \in \mathbb{C}^{N_{1} \times N_{2}}$ implies that $\boldsymbol{A}$ is a complex matrix of dimension $N_{1} \times N_{2} . \boldsymbol{A} \succeq \mathbf{0}$ and $\boldsymbol{A} \succ \mathbf{0}$ imply that $\boldsymbol{A}$ is a positive semidefinite matrix and positive definite matrix, respectively. Identity matrix is denoted by $\boldsymbol{I}$. [.] ${ }^{*}$ denotes complex conjugate transpose operation. $\mathbb{E}[$.$] denotes$ expectation operator. $\|$.$\| denotes 2$-norm operator. Trace of matrix $\boldsymbol{A} \in \mathbb{C}^{N \times N}$ is denoted by $\operatorname{Tr}(\boldsymbol{A})$.

\section{SySTEM MODEL}

We consider full-duplex communication between two users $S_{1}$ and $S_{2}$ in the presence of an eavesdropper $E$. $S_{1}$, $S_{2}$ are assumed to have $M_{1}$ and $M_{2}$ transmit antennas, respectively, and single receive antenna each. $E$ is a passive eavesdropper and it has single receive antenna. The complex channel gains on various links are as shown in Fig. 1, where $\boldsymbol{h}_{11} \in \mathbb{C}^{1 \times M_{1}}, \boldsymbol{h}_{12} \in \mathbb{C}^{1 \times M_{2}}, \boldsymbol{h}_{21} \in \mathbb{C}^{1 \times M_{1}}, \boldsymbol{h}_{22} \in \mathbb{C}^{1 \times M_{2}}$, $\boldsymbol{z}_{1} \in \mathbb{C}^{1 \times M_{1}}$, and $\boldsymbol{z}_{2} \in \mathbb{C}^{1 \times M_{2}}$. $S_{1}$ and $S_{2}$ simultaneously transmit messages $W_{1}$ and $W_{2}$, respectively, in $n$ channel uses. $W_{1}$ and $W_{2}$ are independent and equiprobable over $\left\{1,2, \cdots, 2^{n R_{1}}\right\}$ and $\left\{1,2, \cdots, 2^{n R_{2}}\right\}$, respectively. $R_{1}$ and $R_{2}$ are the information rates (bits per channel use) associated 


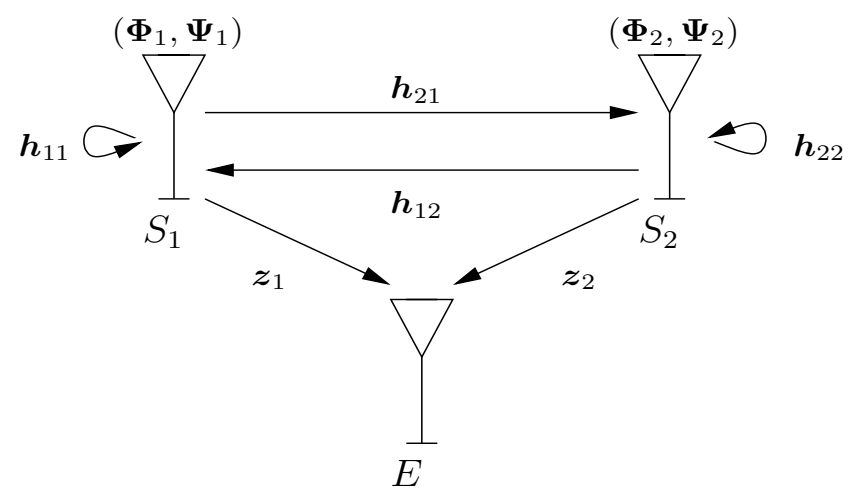

Fig. 1. System model for MISO full-duplex communication. $S_{1}$ has $M_{1}$ transmit antennas and single receive antenna. $S_{2}$ has $M_{2}$ transmit antennas and single receive antenna. $E$ has single receive antenna.

with $W_{1}$ and $W_{2}$, respectively, which need to be transmitted with perfect secrecy with respect to $E$ [15]. $S_{1}$ and $S_{2}$ map $W_{1}$ and $W_{2}$ to codewords $\left\{\boldsymbol{x}_{1 i}\right\}_{i=1}^{n}\left(\boldsymbol{x}_{1 i} \in \mathbb{C}^{M_{1} \times 1}\right.$, i.i.d. $\left.\sim \mathcal{C N}\left(\mathbf{0}, \boldsymbol{\Phi}_{1}\right), \boldsymbol{\Phi}_{1}=\mathbb{E}\left[\boldsymbol{x}_{1 i} \boldsymbol{x}_{1 i}^{*}\right]\right)$ and $\left\{\boldsymbol{x}_{2 i}\right\}_{i=1}^{n}\left(\boldsymbol{x}_{2 i} \in\right.$ $\mathbb{C}^{M_{2} \times 1}$, i.i.d. $\left.\sim \mathcal{C N}\left(\mathbf{0}, \Phi_{2}\right), \boldsymbol{\Phi}_{2}=\mathbb{E}\left[\boldsymbol{x}_{2 i} \boldsymbol{x}_{2 i}^{*}\right]\right)$, respectively, of length $n$. In order to degrade the eavesdropper channels and improve the secrecy rates, both $S_{1}$ and $S_{2}$ inject jamming signals $\left\{\boldsymbol{n}_{1 i}\right\}_{i=1}^{n}\left(\boldsymbol{n}_{1 i} \in \mathbb{C}^{M_{1} \times 1}\right.$, i.i.d. $\sim \mathcal{C N}\left(\mathbf{0}, \boldsymbol{\Psi}_{1}\right)$, $\left.\boldsymbol{\Psi}_{1}=\mathbb{E}\left[\boldsymbol{n}_{1 i} \boldsymbol{n}_{1 i}^{*}\right]\right)$ and $\left\{\boldsymbol{n}_{2 i}\right\}_{i=1}^{n}\left(\boldsymbol{n}_{2 i} \in \mathbb{C}^{M_{2} \times 1}\right.$, i.i.d. $\left.\sim \mathcal{C N}\left(\mathbf{0}, \boldsymbol{\Psi}_{2}\right), \boldsymbol{\Psi}_{2}=\mathbb{E}\left[\boldsymbol{n}_{2 i} \boldsymbol{n}_{2 i}^{*}\right]\right)$, respectively, of length $n$. $S_{1}$ and $S_{2}$ transmit the symbols $\boldsymbol{x}_{1 i}+\boldsymbol{n}_{1 i}$ and $\boldsymbol{x}_{2 i}+\boldsymbol{n}_{2 i}$, respectively, during the $i$ th channel use, $1 \leq i \leq n$. Hereafter, we will denote the symbols in $\left\{\boldsymbol{x}_{1 i}\right\}_{i=1}^{n},\left\{\boldsymbol{x}_{2 i}\right\}_{i=1}^{n}\left\{\boldsymbol{n}_{1 i}\right\}_{i=1}^{n}$, and $\left\{\boldsymbol{n}_{2 i}\right\}_{i=1}^{n}$ by $\boldsymbol{x}_{1}, \boldsymbol{x}_{2}, \boldsymbol{n}_{1}$, and $\boldsymbol{n}_{2}$, respectively. We also assume that all the channel gains remain static over the codeword transmit duration. Let $P_{1}$ and $P_{2}$ be the transmit power budget for $S_{1}$ and $S_{2}$, respectively. This implies that

$$
\operatorname{Tr}\left(\boldsymbol{\Phi}_{1}+\boldsymbol{\Psi}_{1}\right) \leq P_{1}, \quad \operatorname{Tr}\left(\boldsymbol{\Phi}_{2}+\boldsymbol{\Psi}_{2}\right) \leq P_{2} .
$$

Let $y_{1}, y_{2}$, and $y_{E}$ denote the received signals at $S_{1}, S_{2}$ and $E$, respectively. We have

$$
\begin{aligned}
y_{1} & =\boldsymbol{h}_{11}\left(\boldsymbol{x}_{1}+\boldsymbol{n}_{1}\right)+\boldsymbol{h}_{12}\left(\boldsymbol{x}_{2}+\boldsymbol{n}_{2}\right)+\eta_{1}, \\
y_{2} & =\boldsymbol{h}_{21}\left(\boldsymbol{x}_{1}+\boldsymbol{n}_{1}\right)+\boldsymbol{h}_{22}\left(\boldsymbol{x}_{2}+\boldsymbol{n}_{2}\right)+\eta_{2}, \\
y_{E} & =\boldsymbol{z}_{1}\left(\boldsymbol{x}_{1}+\boldsymbol{n}_{1}\right)+\boldsymbol{z}_{2}\left(\boldsymbol{x}_{2}+\boldsymbol{n}_{2}\right)+\eta_{E},
\end{aligned}
$$

where $\eta_{1}, \eta_{2}$, and $\eta_{E}$ are i.i.d. $\left(\sim \mathcal{C N}\left(0, N_{0}\right)\right)$ receiver noise terms.

\section{SUM SECRECY RATE - PERFECT CSI}

In this section, we assume perfect CSI in all the links. Since $S_{1}$ knows the transmitted symbol $\left(\boldsymbol{x}_{1}+\boldsymbol{n}_{1}\right)$, in order to detect $\boldsymbol{x}_{2}, S_{1}$ subtracts $\boldsymbol{h}_{11}\left(\boldsymbol{x}_{1}+\boldsymbol{n}_{1}\right)$ from the received signal $y_{1}$, i.e.,

$$
\begin{aligned}
y_{1}^{\prime} & =y_{1}-\boldsymbol{h}_{11}\left(\boldsymbol{x}_{1}+\boldsymbol{n}_{1}\right) \\
& =\boldsymbol{h}_{12}\left(\boldsymbol{x}_{2}+\boldsymbol{n}_{2}\right)+\eta_{1} .
\end{aligned}
$$

Similarly, since $S_{2}$ knows the transmitted symbol $\left(\boldsymbol{x}_{2}+\boldsymbol{n}_{2}\right)$, to detect $\boldsymbol{x}_{1}, S_{2}$ subtracts $\boldsymbol{h}_{22}\left(\boldsymbol{x}_{2}+\boldsymbol{n}_{2}\right)$ from the received signal $y_{2}$, i.e.,

$$
\begin{aligned}
y_{2}^{\prime} & =y_{2}-\boldsymbol{h}_{22}\left(\boldsymbol{x}_{2}+\boldsymbol{n}_{2}\right) \\
& =\boldsymbol{h}_{21}\left(\boldsymbol{x}_{1}+\boldsymbol{n}_{1}\right)+\eta_{2} .
\end{aligned}
$$

Using (5) and [6), we get the following information rates for $\boldsymbol{x}_{1}$ and $\boldsymbol{x}_{2}$, respectively:

$$
\begin{aligned}
& R_{1}^{\prime} \triangleq I\left(\boldsymbol{x}_{1} ; y_{2}^{\prime}\right)=\log _{2}\left(1+\frac{\boldsymbol{h}_{21} \boldsymbol{\Phi}_{1} \boldsymbol{h}_{21}^{*}}{N_{0}+\boldsymbol{h}_{21} \boldsymbol{\Psi}_{1} \boldsymbol{h}_{21}^{*}}\right), \\
& R_{2}^{\prime} \triangleq I\left(\boldsymbol{x}_{2} ; y_{1}^{\prime}\right)=\log _{2}\left(1+\frac{\boldsymbol{h}_{12} \boldsymbol{\Phi}_{2} \boldsymbol{h}_{12}^{*}}{N_{0}+\boldsymbol{h}_{12} \boldsymbol{\Psi}_{2} \boldsymbol{h}_{12}^{*}}\right) .
\end{aligned}
$$

Using (4), we get the information leakage rate at $E$ as

$$
\begin{aligned}
R_{E}^{\prime} & \triangleq I\left(\boldsymbol{x}_{1}, \boldsymbol{x}_{2} ; y_{E}\right) \\
& =\log _{2}\left(1+\frac{\boldsymbol{z}_{1} \boldsymbol{\Phi}_{1} \boldsymbol{z}_{1}^{*}+\boldsymbol{z}_{2} \boldsymbol{\Phi}_{2} \boldsymbol{z}_{2}^{*}}{N_{0}+\boldsymbol{z}_{1} \boldsymbol{\Psi}_{1} \boldsymbol{z}_{1}^{*}+\boldsymbol{z}_{2} \boldsymbol{\Psi}_{2} \boldsymbol{z}_{2}^{*}}\right) .
\end{aligned}
$$

Using (7), (8), and (9), we get the information capacities $C_{1}^{\prime}$, $C_{2}^{\prime}$, and $C_{E}^{\prime}$, respectively, as follows:

$$
\begin{array}{r}
C_{1}^{\prime}=\log _{2}\left(1+\frac{\left\|\boldsymbol{h}_{21}\right\|^{2} P_{1}}{N_{0}}\right), \\
C_{2}^{\prime}=\log _{2}\left(1+\frac{\left\|\boldsymbol{h}_{12}\right\|^{2} P_{2}}{N_{0}}\right), \\
C_{E}^{\prime}=\log _{2}\left(1+\frac{\left\|\boldsymbol{z}_{1}\right\|^{2} P_{1}+\left\|\boldsymbol{z}_{2}\right\|^{2} P_{2}}{N_{0}}\right) .
\end{array}
$$

A secrecy rate pair $\left(R_{1}, R_{2}\right)$ which falls in the following region is achievable [15]:

$$
\begin{array}{r}
0 \leq R_{1} \leq R_{1}^{\prime}, \quad 0 \leq R_{2} \leq R_{2}^{\prime}, \\
0 \leq R_{1}+R_{2} \leq R_{1}^{\prime}+R_{2}^{\prime}-R_{E}^{\prime}, \\
\boldsymbol{\Phi}_{1} \succeq \mathbf{0}, \quad \boldsymbol{\Psi}_{1} \succeq \mathbf{0}, \quad \operatorname{Tr}\left(\boldsymbol{\Phi}_{1}+\boldsymbol{\Psi}_{1}\right) \leq P_{1}, \\
\boldsymbol{\Phi}_{2} \succeq \mathbf{0}, \quad \boldsymbol{\Psi}_{2} \succeq \mathbf{0}, \quad \operatorname{Tr}\left(\boldsymbol{\Phi}_{2}+\boldsymbol{\Psi}_{2}\right) \leq P_{2} .
\end{array}
$$

We intend to maximize the sum secrecy rate subject to the power constraint, i.e.,

$$
\begin{array}{r}
\max _{\substack{\boldsymbol{\Phi}_{1}, \boldsymbol{\Psi}_{1}^{\prime}, \boldsymbol{\Phi}_{2}, \boldsymbol{\Psi}_{2}}} R_{1}^{\prime}+R_{2}^{\prime}-R_{E}^{\prime} \\
=\max _{\substack{\boldsymbol{\Phi}_{1}, \boldsymbol{\Psi}_{1}, \boldsymbol{\Phi}_{2}, \boldsymbol{\Psi}_{2}}}\left\{\log _{2}\left(1+\frac{\boldsymbol{h}_{21} \boldsymbol{\Phi}_{1} \boldsymbol{h}_{21}^{*}}{N_{0}+\boldsymbol{h}_{21} \boldsymbol{\Psi}_{1} \boldsymbol{h}_{21}^{*}}\right)\right. \\
+\log _{2}\left(1+\frac{\boldsymbol{h}_{12} \boldsymbol{\Phi}_{2} \boldsymbol{h}_{12}^{*}}{N_{0}+\boldsymbol{h}_{12} \boldsymbol{\Psi}_{2} \boldsymbol{h}_{12}^{*}}\right) \\
\left.-\log _{2}\left(1+\frac{\boldsymbol{z}_{1} \boldsymbol{\Phi}_{1} \boldsymbol{z}_{1}^{*}+\boldsymbol{z}_{2} \boldsymbol{\Phi}_{2} \boldsymbol{z}_{2}^{*}}{N_{0}+\boldsymbol{z}_{1} \boldsymbol{\Psi}_{1} \boldsymbol{z}_{1}^{*}+\boldsymbol{z}_{2} \boldsymbol{\Psi}_{2} \boldsymbol{z}_{2}^{*}}\right)\right\}
\end{array}
$$

s.t. $\quad \boldsymbol{\Phi}_{1} \succeq \mathbf{0}, \quad \boldsymbol{\Psi}_{1} \succeq \mathbf{0}, \quad \operatorname{Tr}\left(\boldsymbol{\Phi}_{1}+\boldsymbol{\Psi}_{1}\right) \leq P_{1}$,

$$
\boldsymbol{\Phi}_{2} \succeq \mathbf{0}, \quad \mathbf{\Psi}_{2} \succeq \mathbf{0}, \quad \operatorname{Tr}\left(\boldsymbol{\Phi}_{2}+\boldsymbol{\Psi}_{2}\right) \leq P_{2} .
$$

This is a non-convex optimization problem, and we solve it using two-dimensional search as follows.

Step1 : Divide the intervals $\left[0, C_{1}^{\prime}\right]$ and $\left[0, C_{2}^{\prime}\right]$ in $K$ and $L$ small intervals, respectively, of size $\triangle_{1}=\frac{C_{1}^{\prime}}{K}$ and $\triangle_{2}=$ 
$\frac{C_{2}^{\prime}}{L}$ where $K$ and $L$ are large integers. Let $R_{1}^{\prime k}=k \triangle_{1}$ and $R_{2}^{\prime l}=l \triangle_{2}$, where $k=0,1,2, \cdots, K$ and $l=0,1,2, \cdots, L$.

Step2 : For a given $\left(R_{1}^{\prime k}, R_{2}^{\prime l}\right)$ pair, we minimize $R_{E}^{\prime}$ as follows:

$$
\begin{aligned}
R_{E}^{\prime \prime k l} \triangleq \min _{\substack{\boldsymbol{\Phi}_{1}, \psi_{1}, \boldsymbol{\Phi}_{2}, \psi_{2}}} \log _{2}\left(1+\frac{\boldsymbol{z}_{1} \boldsymbol{\Phi}_{1} \boldsymbol{z}_{1}^{*}+\boldsymbol{z}_{2} \boldsymbol{\Phi}_{2} \boldsymbol{z}_{2}^{*}}{N_{0}+\boldsymbol{z}_{1} \boldsymbol{\Psi}_{1} \boldsymbol{z}_{1}^{*}+\boldsymbol{z}_{2} \boldsymbol{\Psi}_{2} \boldsymbol{z}_{2}^{*}}\right) \\
\text { s.t. } \quad R_{1}^{\prime \prime} k \triangleq \log _{2}\left(1+\frac{\boldsymbol{h}_{21} \boldsymbol{\Phi}_{1} \boldsymbol{h}_{21}^{*}}{N_{0}+\boldsymbol{h}_{21} \boldsymbol{\Psi}_{1} \boldsymbol{h}_{21}^{*}}\right) \geq R_{1}^{\prime k}, \\
R_{2}^{\prime \prime l} \triangleq \log _{2}\left(1+\frac{\boldsymbol{h}_{12} \boldsymbol{\Phi}_{2} \boldsymbol{h}_{12}^{*}}{N_{0}+\boldsymbol{h}_{12} \boldsymbol{\Psi}_{2} \boldsymbol{h}_{12}^{*}}\right) \geq R_{2}^{\prime l}, \\
\boldsymbol{\Phi}_{1} \succeq \mathbf{0}, \quad \boldsymbol{\Psi}_{1} \succeq \mathbf{0}, \quad \operatorname{Tr}\left(\boldsymbol{\Phi}_{1}+\boldsymbol{\Psi}_{1}\right) \leq P_{1}, \\
\boldsymbol{\Phi}_{2} \succeq \mathbf{0}, \quad \mathbf{\Psi}_{2} \succeq \mathbf{0}, \quad \operatorname{Tr}\left(\boldsymbol{\Phi}_{2}+\boldsymbol{\Psi}_{2}\right) \leq P_{2} .
\end{aligned}
$$

The maximum sum secrecy rate is given by $\max _{\substack{k=0,1,2, \ldots, K, l=0,1,2, \ldots, L}}\left(R_{1}^{\prime \prime} k+R_{2}^{\prime \prime l} l-R_{E}^{\prime \prime} k l\right)$. We solve the optimization problem (17) as follows. Dropping the logarithm in the objective function in 177, we rewrite the optimization problem (17) in the following equivalent form:

$$
\min _{t, \mathbf{\Phi}_{1}, \boldsymbol{\Psi}_{1}, \mathbf{\Phi}_{2}, \mathbf{\Psi}_{2}} t
$$

s.t.

$$
\begin{array}{r}
\left(\boldsymbol{z}_{1} \boldsymbol{\Phi}_{1} \boldsymbol{z}_{1}^{*}+\boldsymbol{z}_{2} \boldsymbol{\Phi}_{2} \boldsymbol{z}_{2}^{*}\right)-t\left(N_{0}+\boldsymbol{z}_{1} \boldsymbol{\Psi}_{1} \boldsymbol{z}_{1}^{*}+\boldsymbol{z}_{2} \boldsymbol{\Psi}_{2} \boldsymbol{z}_{2}^{*}\right) \leq 0, \\
\left(2^{R_{1}^{\prime k}}-1\right)\left(N_{0}+\boldsymbol{h}_{21} \boldsymbol{\Psi}_{1} \boldsymbol{h}_{21}^{*}\right)-\left(\boldsymbol{h}_{21} \boldsymbol{\Phi}_{1} \boldsymbol{h}_{21}^{*}\right) \leq 0, \\
\left(2^{R_{2}^{\prime l}}-1\right)\left(N_{0}+\boldsymbol{h}_{12} \boldsymbol{\Psi}_{2} \boldsymbol{h}_{12}^{*}\right)-\left(\boldsymbol{h}_{12} \boldsymbol{\Phi}_{2} \boldsymbol{h}_{12}^{*}\right) \leq 0, \\
\boldsymbol{\Phi}_{1} \succeq \mathbf{0}, \quad \boldsymbol{\Psi}_{1} \succeq \mathbf{0}, \quad \operatorname{Tr}\left(\boldsymbol{\Phi}_{1}+\boldsymbol{\Psi}_{1}\right) \leq P_{1}, \\
\boldsymbol{\Phi}_{2} \succeq \mathbf{0}, \quad \boldsymbol{\Psi}_{2} \succeq \mathbf{0}, \quad \operatorname{Tr}\left(\boldsymbol{\Phi}_{2}+\boldsymbol{\Psi}_{2}\right) \leq P_{2} .
\end{array}
$$

Using the KKT conditions of the above optimization problem, we analyze the ranks of the optimum solutions $\boldsymbol{\Phi}_{1}, \boldsymbol{\Psi}_{1}, \boldsymbol{\Phi}_{2}$, $\Psi_{2}$ in the Appendix. Further, for a given $t$, the above problem is formulated as the following semidefinite feasibility problem [19]:

$$
\text { find } \boldsymbol{\Phi}_{1}, \boldsymbol{\Psi}_{1}, \boldsymbol{\Phi}_{2}, \boldsymbol{\Psi}_{2}
$$

subject to the constraints in 200. The minimum value of $t$, denoted by $t_{m i n}^{k l}$, can be obtained using bisection method [19] as follows. Let $t_{\min }^{k l}$ lie in the interval $\left[t_{\text {lowerlimit }}, t_{\text {upperlimit }}\right]$. The value of $t_{\text {lowerlimit }}$ can be taken as 0 (corresponding to the minimum information rate of 0$)$ and $t_{\text {upperlimit }}$ can be taken as $\left(2^{C_{E}^{\prime}}-1\right)$, which corresponds to the information capacity of the eavesdropper link. Check the feasibility of (21) at $t_{\text {min }}^{k l}=$ $\left(t_{\text {lowerlimit }}+t_{\text {upperlimit }}\right) / 2$. If feasible, then $t_{\text {upperlimit }}=$ $t_{\text {min }}^{k l}$, else $t_{\text {lowerlimit }}=t_{\text {min }}^{k l}$. Repeat this until $t_{\text {upperlimit }}-$ $t_{\text {lowerlimit }} \leq \zeta$, where $\zeta$ is a small positive number. Using $t_{\min }^{k l}$ in (17), $R_{E}^{\prime \prime k l}$ is given by

$$
R_{E}^{\prime \prime k l}=\log _{2}\left(1+t_{m i n}^{k l}\right) .
$$

\section{SUM SECRECY RATE - IMPERFECT CSI}

In this section, we assume that the available CSI in all the links are imperfect [16]-[18], i.e.,

$$
\begin{array}{r}
\boldsymbol{h}_{11}=\boldsymbol{h}_{11}^{0}+\boldsymbol{e}_{11}, \quad \boldsymbol{h}_{12}=\boldsymbol{h}_{12}^{0}+\boldsymbol{e}_{12}, \quad \boldsymbol{h}_{21}=\boldsymbol{h}_{21}^{0}+\boldsymbol{e}_{21}, \\
\boldsymbol{h}_{22}=\boldsymbol{h}_{22}^{0}+\boldsymbol{e}_{22}, \quad \boldsymbol{z}_{1}=\boldsymbol{z}_{1}^{0}+\boldsymbol{e}_{1}, \quad \boldsymbol{z}_{2}=\boldsymbol{z}_{2}^{0}+\boldsymbol{e}_{2},
\end{array}
$$

where $\boldsymbol{h}_{11}^{0}, \boldsymbol{h}_{12}^{0}, \boldsymbol{h}_{21}^{0}, \boldsymbol{h}_{22}^{0}, \boldsymbol{z}_{1}^{0}$, and $\boldsymbol{z}_{2}^{0}$ are the estimates of $\boldsymbol{h}_{11}, \boldsymbol{h}_{12}, \boldsymbol{h}_{21}, \boldsymbol{h}_{22}, \boldsymbol{z}_{1}$, and $\boldsymbol{z}_{2}$, respectively, and $\boldsymbol{e}_{11}, \boldsymbol{e}_{12}$, $\boldsymbol{e}_{21}, \boldsymbol{e}_{22}, \boldsymbol{e}_{1}$, and $\boldsymbol{e}_{2}$ are the corresponding errors. We assume that the norm of the errors are bounded in their respective absolute values as:

$$
\begin{array}{r}
\left\|e_{11}\right\| \leq \epsilon_{11}, \quad\left\|e_{12}\right\| \leq \epsilon_{12}, \quad\left\|e_{21}\right\| \leq \epsilon_{21} \\
\left\|e_{22}\right\| \leq \epsilon_{22}, \quad\left\|e_{1}\right\| \leq \epsilon_{1}, \quad\left\|e_{2}\right\| \leq \epsilon_{2} .
\end{array}
$$

We make the following assumptions with respect to the availability of the CSI at $S_{1}, S_{2}$, and $E$ :

(a.) We assume that only the estimates $\boldsymbol{h}_{11}^{0}, \boldsymbol{h}_{21}^{0}, \boldsymbol{z}_{1}^{0}$, and $\boldsymbol{z}_{2}^{0}$ are available at $S_{1}$ while $\boldsymbol{h}_{12}$ is perfectly known at $S_{1}$ (coherent detection). Similarly, only the estimates $\boldsymbol{h}_{22}^{0}, \boldsymbol{h}_{12}^{0}$, $\boldsymbol{z}_{1}^{0}$, and $\boldsymbol{z}_{2}^{0}$ are available at $S_{2}$ while $\boldsymbol{h}_{21}$ is perfectly known at $S_{2}$ (coherent detection). We assume that $E$ has perfect knowledge of $\boldsymbol{z}_{1}$, and $\boldsymbol{z}_{2}$ (coherent detection). With the above error model, we rewrite (5), (6), and (4) as follows:

$$
\begin{aligned}
y_{1}^{\prime} & =y_{1}-\boldsymbol{h}_{11}^{0}\left(\boldsymbol{x}_{1}+\boldsymbol{n}_{1}\right) \\
& =\boldsymbol{e}_{11}\left(\boldsymbol{x}_{1}+\boldsymbol{n}_{1}\right)+\boldsymbol{h}_{12}\left(\boldsymbol{x}_{2}+\boldsymbol{n}_{2}\right)+\eta_{1}, \\
y_{2}^{\prime} & =y_{2}-\boldsymbol{h}_{22}^{0}\left(\boldsymbol{x}_{2}+\boldsymbol{n}_{2}\right) \\
& =\boldsymbol{h}_{21}\left(\boldsymbol{x}_{1}+\boldsymbol{n}_{1}\right)+\boldsymbol{e}_{22}\left(\boldsymbol{x}_{2}+\boldsymbol{n}_{2}\right)+\eta_{2}, \\
y_{E} & =\boldsymbol{z}_{1}\left(\boldsymbol{x}_{1}+\boldsymbol{n}_{1}\right)+\boldsymbol{z}_{2}\left(\boldsymbol{x}_{2}+\boldsymbol{n}_{2}\right)+\eta_{E} .
\end{aligned}
$$

(b.) We assume that while detecting $\boldsymbol{x}_{2}, S_{1}$ treats the residual term $\boldsymbol{e}_{11}\left(\boldsymbol{x}_{1}+\boldsymbol{n}_{1}\right)$ which appears in (23) as selfnoise. Similarly, while detecting $\boldsymbol{x}_{1}, S_{2}$ treats the residual term $\boldsymbol{e}_{22}\left(\boldsymbol{x}_{2}+\boldsymbol{n}_{2}\right)$ which appears in (24) as self-noise.

Further, in order to compute $R_{1}^{\prime k}, R_{2}^{\prime l}$, and $R_{E}^{\prime \prime} k l$, respectively, as described in Step1 and Step2 in Section 【III we get the worst case capacities $C_{1}^{\prime}, C_{2}^{\prime}$ for $S_{1}, S_{2}$ links, and best case capacity $C_{E}^{\prime}$ for the eavesdropper link with imperfect CSI as follows:

$$
\begin{array}{r}
C_{1}^{\prime}=\log _{2}\left(1+\frac{||\left|\boldsymbol{h}_{21}^{0} \|-\epsilon_{21}\right|^{2} P_{1}}{N_{0}}\right) \text { if } \begin{array}{r}
\left(\left\|\boldsymbol{h}_{21}^{0}\right\|>\epsilon_{21}\right), \\
0 \text { else. }
\end{array} \\
C_{2}^{\prime}=\log _{2}\left(1+\frac{\left|\left\|\boldsymbol{h}_{12}^{0}\right\|-\epsilon_{12}\right|^{2} P_{2}}{N_{0}}\right) \text { if }\left(\begin{array}{r}
\left(\mid \boldsymbol{h}_{12}^{0} \|>\epsilon_{12}\right), \\
0 \text { else. }
\end{array}\right. \\
C_{E}^{\prime}=\log _{2}\left(1+\frac{||\left|\boldsymbol{z}_{1}^{0}\left\|+\left.\epsilon_{1}\right|^{2} P_{1}+|| \boldsymbol{z}_{2}^{0}\right\|+\epsilon_{2}\right|^{2} P_{2}}{N_{0}}\right) .
\end{array}
$$

Using (23), 24), and 25], we write the optimization problem (17) with imperfect CSI as follows:

$$
\begin{array}{r}
R_{E}^{\prime \prime k l} \triangleq \min _{\boldsymbol{\Phi}_{1}, \boldsymbol{\Psi}_{1}, \boldsymbol{\Phi}_{2}, \boldsymbol{\Psi}_{2} \max _{\boldsymbol{e}_{1}, \boldsymbol{e}_{2}} \log _{2}} \\
\left(1+\frac{\left(\boldsymbol{z}_{1}^{0}+\boldsymbol{e}_{1}\right) \boldsymbol{\Phi}_{1}\left(\boldsymbol{z}_{1}^{0}+\boldsymbol{e}_{1}\right)^{*}+\left(\boldsymbol{z}_{2}^{0}+\boldsymbol{e}_{2}\right) \boldsymbol{\Phi}_{2}\left(\boldsymbol{z}_{2}^{0}+\boldsymbol{e}_{2}\right)^{*}}{N_{0}+\left(\boldsymbol{z}_{1}^{0}+\boldsymbol{e}_{1}\right) \boldsymbol{\Psi}_{1}\left(\boldsymbol{z}_{1}^{0}+\boldsymbol{e}_{1}\right)^{*}+\left(\boldsymbol{z}_{2}^{0}+\boldsymbol{e}_{2}\right) \boldsymbol{\Psi}_{2}\left(\boldsymbol{z}_{2}^{0}+\boldsymbol{e}_{2}\right)^{*}}\right)
\end{array}
$$




$$
\begin{aligned}
& \text { s.t. } \quad R_{1}^{\prime \prime k} \triangleq \min _{e_{21}, e_{22}} \log _{2} \\
& \left(1+\frac{\left(\boldsymbol{h}_{21}^{0}+\boldsymbol{e}_{21}\right) \boldsymbol{\Phi}_{1}\left(\boldsymbol{h}_{21}^{0}+\boldsymbol{e}_{21}\right)^{*}}{N_{0}+\boldsymbol{e}_{22}\left(\boldsymbol{\Phi}_{2}+\boldsymbol{\Psi}_{2}\right) \boldsymbol{e}_{22}^{*}+\left(\boldsymbol{h}_{21}^{0}+\boldsymbol{e}_{21}\right) \boldsymbol{\Psi}_{1}\left(\boldsymbol{h}_{21}^{0}+\boldsymbol{e}_{21}\right)^{*}}\right) \\
& \geq R_{1}^{\prime k} \\
& R_{2}^{\prime \prime l} \triangleq \min _{e_{11}, e_{12}} \log _{2} \\
& \left(1+\frac{\left(\boldsymbol{h}_{12}^{0}+\boldsymbol{e}_{12}\right) \boldsymbol{\Phi}_{2}\left(\boldsymbol{h}_{12}^{0}+\boldsymbol{e}_{12}\right)^{*}}{N_{0}+\boldsymbol{e}_{11}\left(\boldsymbol{\Phi}_{1}+\boldsymbol{\Psi}_{1}\right) \boldsymbol{e}_{11}^{*}+\left(\boldsymbol{h}_{12}^{0}+\boldsymbol{e}_{12}\right) \boldsymbol{\Psi}_{2}\left(\boldsymbol{h}_{12}^{0}+\boldsymbol{e}_{12}\right)^{*}}\right) \\
& \geq R_{2}^{\prime l} \\
& \left\|\boldsymbol{e}_{11}\right\|^{2} \leq \epsilon_{11}^{2}, \quad\left\|\boldsymbol{e}_{12}\right\|^{2} \leq \epsilon_{12}^{2}, \quad\left\|\boldsymbol{e}_{21}\right\|^{2} \leq \epsilon_{21}^{2}, \\
& \left\|\boldsymbol{e}_{22}\right\|^{2} \leq \epsilon_{22}^{2}, \quad\left\|\boldsymbol{e}_{1}\right\|^{2} \leq \epsilon_{1}^{2}, \quad\left\|\boldsymbol{e}_{2}\right\|^{2} \leq \epsilon_{2}^{2}, \\
& \boldsymbol{\Phi}_{1} \succeq \mathbf{0}, \quad \boldsymbol{\Psi}_{1} \succeq \mathbf{0}, \quad \operatorname{Tr}\left(\boldsymbol{\Phi}_{1}+\boldsymbol{\Psi}_{1}\right) \leq P_{1}, \\
& \boldsymbol{\Phi}_{2} \succeq \mathbf{0}, \quad \boldsymbol{\Psi}_{2} \succeq \mathbf{0}, \quad \operatorname{Tr}\left(\boldsymbol{\Phi}_{2}+\boldsymbol{\Psi}_{2}\right) \leq P_{2}
\end{aligned}
$$

In the constraints (30) and 31, additional noise appear due the terms $\boldsymbol{e}_{22}\left(\boldsymbol{x}_{2}+\boldsymbol{n}_{2}\right)$ and $\boldsymbol{e}_{11}\left(\boldsymbol{x}_{1}+\boldsymbol{n}_{1}\right)$, respectively, which have been treated as self noise.

We solve the optimization problem (29) as follows. Dropping the logarithm in the objective function in 29], we write the optimization problem (29) in the following equivalent form:

$$
\left.\begin{array}{r}
\min _{\boldsymbol{\Phi}_{1}, \boldsymbol{\Phi}_{2}, \boldsymbol{\Psi}_{2}} \max _{\boldsymbol{e}_{1}, \boldsymbol{e}_{2}} \\
\left(\boldsymbol{z}_{1}^{0}+\boldsymbol{e}_{1}\right) \boldsymbol{\Phi}_{1}\left(\boldsymbol{z}_{1}^{0}+\boldsymbol{e}_{1}\right)^{*}+\left(\boldsymbol{z}_{2}^{0}+\boldsymbol{e}_{2}\right) \boldsymbol{\Phi}_{2}\left(\boldsymbol{z}_{2}^{0}+\boldsymbol{e}_{2}\right)^{*} \\
\hline N_{0}+\left(\boldsymbol{z}_{1}^{0}+\boldsymbol{e}_{1}\right) \boldsymbol{\Psi}_{1}\left(\boldsymbol{z}_{1}^{0}+\boldsymbol{e}_{1}\right)^{*}+\left(\boldsymbol{z}_{2}^{0}+\boldsymbol{e}_{2}\right) \boldsymbol{\Psi}_{2}\left(\boldsymbol{z}_{2}^{0}+\boldsymbol{e}_{2}\right)^{*}
\end{array}\right)
$$

Solving the above optimization problem is hard due to the presence of $e_{1}$ and $e_{2}$ in both the numerator and denominator of the objective function in 347. Similarly, $e_{21}$ and $e_{12}$ appear in both the numerator and denominator of the constraints in (35) and (36), respectively. By independently constraining the various quadratic terms appearing in the objective function in (34) and the constraints (35) and (36), we get the following upper bound for the above optimization problem:

$$
\begin{array}{r}
\min _{\boldsymbol{\Phi}_{1}, \boldsymbol{\Psi}_{1}, \mathbf{\Phi}_{2}, \boldsymbol{\Psi}_{2}} \min _{t_{1}, t_{2}, \cdots, t_{10}}\left(\frac{t_{1}+t_{2}}{N_{0}+t_{3}+t_{4}}\right) \\
\text { s.t. } t_{3} \geq 0, t_{4} \geq 0, t_{5} \geq 0, t_{8} \geq 0, \\
\forall \boldsymbol{e}_{1} \quad \text { s.t. }\left\|\boldsymbol{e}_{1}\right\|^{2} \leq \epsilon_{1}^{2} \Longrightarrow \\
\left(\boldsymbol{z}_{1}^{0}+\boldsymbol{e}_{1}\right) \boldsymbol{\Phi}_{1}\left(\boldsymbol{z}_{1}^{0}+\boldsymbol{e}_{1}\right)^{*}-t_{1} \leq 0, \\
\forall \boldsymbol{e}_{1} \quad \text { s.t. }\left\|\boldsymbol{e}_{1}\right\|^{2} \leq \epsilon_{1}^{2} \Longrightarrow
\end{array}
$$

$$
\begin{aligned}
& -\left(\boldsymbol{z}_{1}^{0}+\boldsymbol{e}_{1}\right) \boldsymbol{\Psi}_{1}\left(\boldsymbol{z}_{1}^{0}+\boldsymbol{e}_{1}\right)^{*}+t_{3} \leq 0, \\
& \forall e_{2} \quad \text { s.t. }\left\|\boldsymbol{e}_{2}\right\|^{2} \leq \epsilon_{2}^{2} \Longrightarrow \\
& \left(\boldsymbol{z}_{2}^{0}+\boldsymbol{e}_{2}\right) \boldsymbol{\Phi}_{2}\left(\boldsymbol{z}_{2}^{0}+\boldsymbol{e}_{2}\right)^{*}-t_{2} \leq 0, \\
& \forall e_{2} \quad \text { s.t. }\left\|e_{2}\right\|^{2} \leq \epsilon_{2}^{2} \Longrightarrow \\
& -\left(\boldsymbol{z}_{2}^{0}+\boldsymbol{e}_{2}\right) \boldsymbol{\Psi}_{2}\left(\boldsymbol{z}_{2}^{0}+\boldsymbol{e}_{2}\right)^{*}+t_{4} \leq 0 \text {, } \\
& \left(\frac{t_{5}}{N_{0}+t_{6}+t_{7}}\right) \geq\left(2^{R_{1}^{\prime k}}-1\right), \\
& \forall e_{21} \quad \text { s.t. }\left\|e_{21}\right\|^{2} \leq \epsilon_{21}^{2} \Longrightarrow \\
& -\left(\boldsymbol{h}_{21}^{0}+\boldsymbol{e}_{21}\right) \boldsymbol{\Phi}_{1}\left(\boldsymbol{h}_{21}^{0}+\boldsymbol{e}_{21}\right)^{*}+t_{5} \leq 0 \text {, } \\
& \forall e_{21} \quad \text { s.t. }\left\|e_{21}\right\|^{2} \leq \epsilon_{21}^{2} \Longrightarrow \\
& \left(\boldsymbol{h}_{21}^{0}+\boldsymbol{e}_{21}\right) \boldsymbol{\Psi}_{1}\left(\boldsymbol{h}_{21}^{0}+\boldsymbol{e}_{21}\right)^{*}-t_{7} \leq 0, \\
& \forall e_{22} \quad \text { s.t. } \quad\left\|e_{22}\right\|^{2} \leq \epsilon_{22}^{2} \Longrightarrow \\
& \boldsymbol{e}_{22}\left(\boldsymbol{\Phi}_{2}+\boldsymbol{\Psi}_{2}\right) \boldsymbol{e}_{22}^{*}-t_{6} \leq 0, \\
& \left(\frac{t_{8}}{N_{0}+t_{9}+t_{10}}\right) \geq\left(2^{R_{2}^{\prime}}-1\right), \\
& \forall e_{12} \quad \text { s.t. }\left\|e_{12}\right\|^{2} \leq \epsilon_{12}^{2} \Longrightarrow \\
& -\left(\boldsymbol{h}_{12}^{0}+\boldsymbol{e}_{12}\right) \boldsymbol{\Phi}_{2}\left(\boldsymbol{h}_{12}^{0}+\boldsymbol{e}_{12}\right)^{*}+t_{8} \leq 0, \\
& \forall e_{12} \quad \text { s.t. }\left\|e_{12}\right\|^{2} \leq \epsilon_{12}^{2} \Longrightarrow \\
& \left(\boldsymbol{h}_{12}^{0}+\boldsymbol{e}_{12}\right) \boldsymbol{\Psi}_{2}\left(\boldsymbol{h}_{12}^{0}+\boldsymbol{e}_{12}\right)^{*}-t_{10} \leq 0 \text {, } \\
& \forall e_{11} \quad \text { s.t. } \quad\left\|e_{11}\right\|^{2} \leq \epsilon_{11}^{2} \Longrightarrow \\
& \boldsymbol{e}_{11}\left(\boldsymbol{\Phi}_{1}+\boldsymbol{\Psi}_{1}\right) \boldsymbol{e}_{11}^{*}-t_{9} \leq 0, \\
& \boldsymbol{\Phi}_{1} \succeq \mathbf{0}, \quad \boldsymbol{\Psi}_{1} \succeq \mathbf{0}, \quad \operatorname{Tr}\left(\boldsymbol{\Phi}_{1}+\boldsymbol{\Psi}_{1}\right) \leq P_{1}, \\
& \boldsymbol{\Phi}_{2} \succeq \mathbf{0}, \quad \boldsymbol{\Psi}_{2} \succeq \mathbf{0}, \quad \operatorname{Tr}\left(\boldsymbol{\Phi}_{2}+\boldsymbol{\Psi}_{2}\right) \leq P_{2}
\end{aligned}
$$

We use the S-procedure to transform the pairs of quadratic inequalities in 40, 44, 42), 43, 44, 46, 47, 49, (50), and (51) to equivalent linear matrix inequalities (LMI) [19]. With this, we get the following single minimization form for the above optimization problem:

$$
\begin{aligned}
& \min _{\substack{\mathbf{\Phi}_{1}, \boldsymbol{\Psi}_{1}, \boldsymbol{\Phi}_{2}, \boldsymbol{\Psi}_{2}, t_{1}, t_{2}, \cdots, t_{10}, \lambda_{1}, \lambda_{2}, \cdots, \lambda_{10}, t}} t \\
& \text { s.t. } t_{3} \geq 0, t_{4} \geq 0, t_{5} \geq 0, t_{8} \geq 0 \\
& \left(t_{1}+t_{2}\right)-t\left(N_{0}+t_{3}+t_{4}\right) \leq 0, \\
& \left(2^{R_{1}^{\prime} k}-1\right)\left(N_{0}+t_{6}+t_{7}\right)-t_{5} \leq 0, \\
& \left(2^{R_{2}^{\prime} l}-1\right)\left(N_{0}+t_{9}+t_{10}\right)-t_{8} \leq 0 \text {, } \\
& {\left[\begin{array}{cc}
-\boldsymbol{\Phi}_{1}+\lambda_{1} \boldsymbol{I} & -\boldsymbol{\Phi}_{1} \boldsymbol{z}_{1}^{0 *} \\
-\boldsymbol{z}_{1}^{0} \boldsymbol{\Phi}_{1}^{*} & -\boldsymbol{z}_{1}^{0} \boldsymbol{\Phi}_{1} \boldsymbol{z}_{1}^{0 *}+t_{1}-\lambda_{1} \epsilon_{1}^{2}
\end{array}\right] \succeq \mathbf{0}, \quad \lambda_{1} \geq 0,} \\
& {\left[\begin{array}{cc}
\boldsymbol{\Psi}_{1}+\lambda_{2} \boldsymbol{I} & \boldsymbol{\Psi}_{1} \boldsymbol{z}_{1}^{0 *} \\
\boldsymbol{z}_{1}^{0} \boldsymbol{\Psi}_{1}^{*} & \boldsymbol{z}_{1}^{0} \boldsymbol{\Psi}_{1} \boldsymbol{z}_{1}^{0 *}-t_{3}-\lambda_{2} \epsilon_{1}^{2}
\end{array}\right] \succeq \mathbf{0}, \quad \lambda_{2} \geq 0,} \\
& {\left[\begin{array}{cc}
-\boldsymbol{\Phi}_{2}+\lambda_{3} \boldsymbol{I} & -\boldsymbol{\Phi}_{2} \boldsymbol{z}_{2}^{0 *} \\
-\boldsymbol{z}_{2}^{0} \boldsymbol{\Phi}_{2}^{*} & -\boldsymbol{z}_{2}^{0} \boldsymbol{\Phi}_{2} \boldsymbol{z}_{2}^{0 *}+t_{2}-\lambda_{3} \epsilon_{2}^{2}
\end{array}\right] \succeq \mathbf{0}, \quad \lambda_{3} \geq 0,} \\
& {\left[\begin{array}{cc}
\boldsymbol{\Psi}_{2}+\lambda_{4} \boldsymbol{I} & \boldsymbol{\Psi}_{2} \boldsymbol{z}_{2}^{0 *} \\
\boldsymbol{z}_{2}^{0} \boldsymbol{\Psi}_{2}^{*} & \boldsymbol{z}_{2}^{0} \boldsymbol{\Psi}_{2} \boldsymbol{z}_{2}^{0 *}-t_{4}-\lambda_{4} \epsilon_{2}^{2}
\end{array}\right] \succeq \mathbf{0}, \quad \lambda_{4} \geq 0,} \\
& {\left[\begin{array}{cc}
\boldsymbol{\Phi}_{1}+\lambda_{5} \boldsymbol{I} & \boldsymbol{\Phi}_{1} \boldsymbol{h}_{21}^{0 *} \\
\boldsymbol{h}_{21}^{0} \boldsymbol{\Phi}_{1}^{*} & \boldsymbol{h}_{21}^{0} \boldsymbol{\Phi}_{1} \boldsymbol{h}_{21}^{0 *}-t_{5}-\lambda_{5} \epsilon_{21}^{2}
\end{array}\right] \succeq \mathbf{0}, \quad \lambda_{5} \geq 0,} \\
& {\left[\begin{array}{cc}
-\boldsymbol{\Psi}_{1}+\lambda_{6} \boldsymbol{I} & -\boldsymbol{\Psi}_{1} \boldsymbol{h}_{21}^{0 *} \\
-\boldsymbol{h}_{21}^{0} \boldsymbol{\Psi}_{1}^{*} & -\boldsymbol{h}_{21}^{0} \boldsymbol{\Psi}_{1} \boldsymbol{h}_{21}^{0 *}+t_{7}-\lambda_{6} \epsilon_{21}^{2}
\end{array}\right] \succeq \mathbf{0}, \quad \lambda_{6} \geq 0,}
\end{aligned}
$$




$$
\begin{aligned}
& {\left[\begin{array}{cc}
-\left(\boldsymbol{\Phi}_{2}+\mathbf{\Psi}_{2}\right)+\lambda_{7} \boldsymbol{I} & \mathbf{0} \\
\mathbf{0} & t_{6}-\lambda_{7} \epsilon_{22}^{2}
\end{array}\right] \succeq \mathbf{0}, \quad \lambda_{7} \geq 0,} \\
& {\left[\begin{array}{cc}
\boldsymbol{\Phi}_{2}+\lambda_{8} \boldsymbol{I} & \boldsymbol{\Phi}_{2} \boldsymbol{h}_{12}^{0 *} \\
\boldsymbol{h}_{12}^{0} \boldsymbol{\Phi}_{2}^{*} & \boldsymbol{h}_{12}^{0} \boldsymbol{\Phi}_{2} \boldsymbol{h}_{12}^{0 *}-t_{8}-\lambda_{8} \epsilon_{12}^{2}
\end{array}\right] \succeq \mathbf{0}, \quad \lambda_{8} \geq 0,} \\
& {\left[\begin{array}{cc}
-\boldsymbol{\Psi}_{2}+\lambda_{9} \boldsymbol{I} & -\boldsymbol{\Psi}_{2} \boldsymbol{h}_{12}^{0 *} \\
-\boldsymbol{h}_{12}^{0} \boldsymbol{\Psi}_{2}^{*} & -\boldsymbol{h}_{12}^{0} \boldsymbol{\Psi}_{2} \boldsymbol{h}_{12}^{0 *}+t_{10}-\lambda_{9} \epsilon_{12}^{2}
\end{array}\right] \succeq \mathbf{0}, \quad \lambda_{9} \geq 0,} \\
& {\left[\begin{array}{cc}
-\left(\boldsymbol{\Phi}_{1}+\boldsymbol{\Psi}_{1}\right)+\lambda_{10} \boldsymbol{I} & \mathbf{0} \\
\mathbf{0} & t_{9}-\lambda_{10} \epsilon_{11}^{2}
\end{array}\right] \succeq \mathbf{0}, \quad \lambda_{10} \geq 0,} \\
& \boldsymbol{\Phi}_{1} \succeq \mathbf{0}, \quad \boldsymbol{\Psi}_{1} \succeq \mathbf{0}, \quad \operatorname{Tr}\left(\boldsymbol{\Phi}_{1}+\boldsymbol{\Psi}_{1}\right) \leq P_{1}, \\
& \boldsymbol{\Phi}_{2} \succeq \mathbf{0}, \quad \boldsymbol{\Psi}_{2} \succeq \mathbf{0}, \quad \operatorname{Tr}\left(\boldsymbol{\Phi}_{2}+\boldsymbol{\Psi}_{2}\right) \leq P_{2} .
\end{aligned}
$$

For a given $t$, the above problem is formulated as the following semidefinite feasibility problem [19]:

$$
\text { find } \boldsymbol{\Phi}_{1}, \boldsymbol{\Psi}_{1}, \boldsymbol{\Phi}_{2}, \boldsymbol{\Psi}_{2}, t_{1}, \cdots, t_{10}, \lambda_{1}, \cdots, \lambda_{10},
$$

subject to the constraints in (54). The minimum value of $t$, denoted by $t_{m i n}^{k l}$, can be obtained using bisection method [19] as described in Section [III The value of $t_{\text {lowerlimit }}$ can be taken as 0 (corresponding to the minimum information rate of 0 ). The value of $t_{\text {upperlimit }}$ can be taken as $\left(2^{C_{E}^{\prime}}-1\right)$, which corresponds to the best case information capacity of the eavesdropper link. Using $t_{m i n}^{k l}$ in (29), the upper bound on $R_{E}^{\prime \prime k l}$ is given by

$$
R_{E}^{\prime \prime k l} \leq \log _{2}\left(1+t_{m i n}^{k l}\right) .
$$

Similarly, denoting the optimal values of $t_{5}, \cdots, t_{10}$ by $t_{5}^{k l}, \cdots, t_{10}^{k l}$, we obtain lower bounds on $R_{1}^{\prime \prime} k$ and $R_{2}^{\prime \prime l}$ as

$$
\begin{gathered}
R_{1}^{\prime \prime k} \geq \log _{2}\left(1+\frac{t_{5}^{k l}}{N_{0}+t_{6}^{k l}+t_{7}^{k l}}\right), \\
R_{2}^{\prime \prime l} \geq \log _{2}\left(1+\frac{t_{8}^{k l}}{N_{0}+t_{9}^{k l}+t_{10}^{k l}}\right) .
\end{gathered}
$$

Using the upper bound from 56 and lower bounds from 57 and (58), the lower bound on the worst case sum secrecy rate is given by $\max _{\substack{k=0,1,2, \ldots, K \\ l=0,1,2, \ldots, L_{L}}}\left(R_{1}^{\prime \prime} k+R_{2}^{\prime \prime l}-R_{E}^{\prime \prime} k l\right)$.

Remark: We note that when $S_{1}$ and $S_{2}$ do not transmit jamming signals, the optimization problems (34) and 38 will be equivalent, and the sum secrecy rate will be exact. However, the lower bound on the sum secrecy rate as obtained above with jamming strategies will always be greater than or equal to the (exact) sum secrecy rate with no jamming strategies.

\section{A. Transmit Power Minimization with SINR Constraints}

In this subsection, we minimize the total transmit power (i.e., $S_{1}$ transmit power plus $S_{2}$ transmit power) with imperfect CSI subject to receive SINR constraints at $S_{1}, S_{2}, E$, and individual transmit power constraints. The optimization problem to minimize the total transmit power is as follows:

$$
\begin{aligned}
& \min _{\boldsymbol{\Phi}_{1}, \boldsymbol{\Psi}_{1}, \boldsymbol{\Phi}_{2}, \boldsymbol{\Psi}_{2}} \operatorname{Tr}\left(\boldsymbol{\Phi}_{1}+\boldsymbol{\Psi}_{1}\right)+\operatorname{Tr}\left(\boldsymbol{\Phi}_{2}+\boldsymbol{\Psi}_{2}\right) \\
& \text { s.t. } \quad \max _{e_{1}, e_{2}} \\
& \left(\frac{\left(\boldsymbol{z}_{1}^{0}+\boldsymbol{e}_{1}\right) \boldsymbol{\Phi}_{1}\left(\boldsymbol{z}_{1}^{0}+\boldsymbol{e}_{1}\right)^{*}+\left(\boldsymbol{z}_{2}^{0}+\boldsymbol{e}_{2}\right) \boldsymbol{\Phi}_{2}\left(\boldsymbol{z}_{2}^{0}+\boldsymbol{e}_{2}\right)^{*}}{N_{0}+\left(\boldsymbol{z}_{1}^{0}+\boldsymbol{e}_{1}\right) \boldsymbol{\Psi}_{1}\left(\boldsymbol{z}_{1}^{0}+\boldsymbol{e}_{1}\right)^{*}+\left(\boldsymbol{z}_{2}^{0}+\boldsymbol{e}_{2}\right) \boldsymbol{\Psi}_{2}\left(\boldsymbol{z}_{2}^{0}+\boldsymbol{e}_{2}\right)^{*}}\right) \\
& \leq \gamma_{E} \\
& \min _{\boldsymbol{e}_{21}, \boldsymbol{e}_{22}} \\
& \left(\frac{\left(\boldsymbol{h}_{21}^{0}+\boldsymbol{e}_{21}\right) \boldsymbol{\Phi}_{1}\left(\boldsymbol{h}_{21}^{0}+\boldsymbol{e}_{21}\right)^{*}}{N_{0}+\boldsymbol{e}_{22}\left(\boldsymbol{\Phi}_{2}+\boldsymbol{\Psi}_{2}\right) \boldsymbol{e}_{22}^{*}+\left(\boldsymbol{h}_{21}^{0}+\boldsymbol{e}_{21}\right) \boldsymbol{\Psi}_{1}\left(\boldsymbol{h}_{21}^{0}+\boldsymbol{e}_{21}\right)^{*}}\right) \\
& \geq \gamma_{S_{2}} \\
& \min _{\boldsymbol{e}_{11}, \boldsymbol{e}_{12}} \\
& \left(\frac{\left(\boldsymbol{h}_{12}^{0}+\boldsymbol{e}_{12}\right) \boldsymbol{\Phi}_{2}\left(\boldsymbol{h}_{12}^{0}+\boldsymbol{e}_{12}\right)^{*}}{N_{0}+\boldsymbol{e}_{11}\left(\boldsymbol{\Phi}_{1}+\boldsymbol{\Psi}_{1}\right) \boldsymbol{e}_{11}^{*}+\left(\boldsymbol{h}_{12}^{0}+\boldsymbol{e}_{12}\right) \boldsymbol{\Psi}_{2}\left(\boldsymbol{h}_{12}^{0}+\boldsymbol{e}_{12}\right)^{*}}\right) \\
& \geq \gamma_{S_{1}} \text {, } \\
& \left\|e_{11}\right\|^{2} \leq \epsilon_{11}^{2}, \quad\left\|e_{12}\right\|^{2} \leq \epsilon_{12}^{2}, \quad\left\|e_{21}\right\|^{2} \leq \epsilon_{21}^{2}, \\
& \left\|\boldsymbol{e}_{22}\right\|^{2} \leq \epsilon_{22}^{2}, \quad\left\|\boldsymbol{e}_{1}\right\|^{2} \leq \epsilon_{1}^{2}, \quad\left\|\boldsymbol{e}_{2}\right\|^{2} \leq \epsilon_{2}^{2}, \\
& \mathbf{\Phi}_{1} \succeq \mathbf{0}, \quad \boldsymbol{\Psi}_{1} \succeq \mathbf{0}, \quad \operatorname{Tr}\left(\boldsymbol{\Phi}_{1}+\boldsymbol{\Psi}_{1}\right) \leq P_{1}, \\
& \boldsymbol{\Phi}_{2} \succeq \mathbf{0}, \quad \mathbf{\Psi}_{2} \succeq \mathbf{0}, \quad \operatorname{Tr}\left(\boldsymbol{\Phi}_{2}+\boldsymbol{\Psi}_{2}\right) \leq P_{2} .
\end{aligned}
$$

The left hand side of the inequality in the constraint 60 corresponds to the best case received SINR at the eavesdropper over the region of CSI error uncertainty. Similarly, the left hand side of the inequality in the constraints 61 and 62 correspond to the worst case received SINR at $S_{2}$, and $S_{1}$, respectively. $\gamma_{E}, \gamma_{S_{2}}$, and $\gamma_{S_{1}}$ are known SINR thresholds at $E, S_{2}$, and $S_{1}$, respectively. Solving the above optimization problem is hard due to the presence of $e_{1}$ and $e_{2}$ in both the numerator and denominator of the SINR expression of the eavesdropper in 60). Similarly, $e_{21}$ and $e_{12}$ appear in both the numerator and denominator of the SINR expressions of $S_{2}$ and $S_{1}$ in the constraints (61) and (62), respectively. By independently constraining the various quadratic terms appearing in the constraints (60), 61), and 662), and further using the S-procedure, we get the following upper bound for the above optimization problem:

$$
\begin{aligned}
& \min _{\substack{\boldsymbol{\Phi}_{1}, \boldsymbol{\Psi}_{1}, \mathbf{\Phi}_{2}, \boldsymbol{\Psi}_{2}, t_{1}, t_{2}, \ldots, t_{10}, \lambda_{1}, \lambda_{2}, \ldots, \lambda_{10}}} \operatorname{Tr}\left(\boldsymbol{\Phi}_{1}+\mathbf{\Psi}_{1}\right)+\operatorname{Tr}\left(\boldsymbol{\Phi}_{2}+\mathbf{\Psi}_{2}\right) \\
& \text { s.t. } t_{3} \geq 0, t_{4} \geq 0, t_{5} \geq 0, t_{8} \geq 0 \text {, } \\
& \left(t_{1}+t_{2}\right)-\gamma_{E}\left(N_{0}+t_{3}+t_{4}\right) \leq 0, \\
& \gamma_{S_{2}}\left(N_{0}+t_{6}+t_{7}\right)-t_{5} \leq 0 \text {, } \\
& \gamma_{S_{1}}\left(N_{0}+t_{9}+t_{10}\right)-t_{8} \leq 0 \text {, } \\
& {\left[\begin{array}{cc}
-\boldsymbol{\Phi}_{1}+\lambda_{1} \boldsymbol{I} & -\boldsymbol{\Phi}_{1} \boldsymbol{z}_{1}^{0 *} \\
-\boldsymbol{z}_{1}^{0} \boldsymbol{\Phi}_{1}^{*} & -\boldsymbol{z}_{1}^{0} \boldsymbol{\Phi}_{1} \boldsymbol{z}_{1}^{0 *}+t_{1}-\lambda_{1} \epsilon_{1}^{2}
\end{array}\right] \succeq \mathbf{0}, \quad \lambda_{1} \geq 0,} \\
& {\left[\begin{array}{cc}
\boldsymbol{\Psi}_{1}+\lambda_{2} \boldsymbol{I} & \boldsymbol{\Psi}_{1} \boldsymbol{z}_{1}^{0 *} \\
\boldsymbol{z}_{1}^{0} \boldsymbol{\Psi}_{1}^{*} & \boldsymbol{z}_{1}^{0} \boldsymbol{\Psi}_{1} \boldsymbol{z}_{1}^{0 *}-t_{3}-\lambda_{2} \epsilon_{1}^{2}
\end{array}\right] \succeq \mathbf{0}, \quad \lambda_{2} \geq 0,} \\
& {\left[\begin{array}{cc}
-\boldsymbol{\Phi}_{2}+\lambda_{3} \boldsymbol{I} & -\boldsymbol{\Phi}_{2} \boldsymbol{z}_{2}^{0 *} \\
-\boldsymbol{z}_{2}^{0} \boldsymbol{\Phi}_{2}^{*} & -\boldsymbol{z}_{2}^{0} \boldsymbol{\Phi}_{2} \boldsymbol{z}_{2}^{0 *}+t_{2}-\lambda_{3} \epsilon_{2}^{2}
\end{array}\right] \succeq \mathbf{0}, \quad \lambda_{3} \geq 0,} \\
& {\left[\begin{array}{cc}
\boldsymbol{\Psi}_{2}+\lambda_{4} \boldsymbol{I} & \boldsymbol{\Psi}_{2} \boldsymbol{z}_{2}^{0 *} \\
\boldsymbol{z}_{2}^{0} \boldsymbol{\Psi}_{2}^{*} & \boldsymbol{z}_{2}^{0} \boldsymbol{\Psi}_{2} \boldsymbol{z}_{2}^{0 *}-t_{4}-\lambda_{4} \epsilon_{2}^{2}
\end{array}\right] \succeq \mathbf{0}, \quad \lambda_{4} \geq 0,} \\
& {\left[\begin{array}{cc}
\boldsymbol{\Phi}_{1}+\lambda_{5} \boldsymbol{I} & \boldsymbol{\Phi}_{1} \boldsymbol{h}_{21}^{0 *} \\
\boldsymbol{h}_{21}^{0} \boldsymbol{\Phi}_{1}^{*} & \boldsymbol{h}_{21}^{0} \boldsymbol{\Phi}_{1} \boldsymbol{h}_{21}^{0 *}-t_{5}-\lambda_{5} \epsilon_{21}^{2}
\end{array}\right] \succeq \mathbf{0}, \quad \lambda_{5} \geq 0,}
\end{aligned}
$$




$$
\begin{aligned}
& {\left[\begin{array}{cc}
-\boldsymbol{\Psi}_{1}+\lambda_{6} \boldsymbol{I} & -\boldsymbol{\Psi}_{1} \boldsymbol{h}_{21}^{0 *} \\
-\boldsymbol{h}_{21}^{0} \boldsymbol{\Psi}_{1}^{*} & -\boldsymbol{h}_{21}^{0} \boldsymbol{\Psi}_{1} \boldsymbol{h}_{21}^{0 *}+t_{7}-\lambda_{6} \epsilon_{21}^{2}
\end{array}\right] \succeq \mathbf{0}, \quad \lambda_{6} \geq 0,} \\
& {\left[\begin{array}{cc}
-\left(\boldsymbol{\Phi}_{2}+\boldsymbol{\Psi}_{2}\right)+\lambda_{7} \boldsymbol{I} & \mathbf{0} \\
\mathbf{0} & t_{6}-\lambda_{7} \epsilon_{22}^{2}
\end{array}\right] \succeq \mathbf{0}, \quad \lambda_{7} \geq 0,} \\
& {\left[\begin{array}{cc}
\boldsymbol{\Phi}_{2}+\lambda_{8} \boldsymbol{I} & \boldsymbol{\Phi}_{2} \boldsymbol{h}_{12}^{0 *} \\
\boldsymbol{h}_{12}^{0} \boldsymbol{\Phi}_{2}^{*} & \boldsymbol{h}_{12}^{0} \boldsymbol{\Phi}_{2} \boldsymbol{h}_{12}^{0 *}-t_{8}-\lambda_{8} \epsilon_{12}^{2}
\end{array}\right] \succeq \mathbf{0}, \quad \lambda_{8} \geq 0,} \\
& {\left[\begin{array}{cc}
-\boldsymbol{\Psi}_{2}+\lambda_{9} \boldsymbol{I} & -\boldsymbol{\Psi}_{2} \boldsymbol{h}_{12}^{0 *} \\
-\boldsymbol{h}_{12}^{0} \boldsymbol{\Psi}_{2}^{*} & -\boldsymbol{h}_{12}^{0} \boldsymbol{\Psi}_{2} \boldsymbol{h}_{12}^{0 *}+t_{10}-\lambda_{9} \epsilon_{12}^{2}
\end{array}\right] \succeq \mathbf{0}, \quad \lambda_{9} \geq 0,} \\
& {\left[\begin{array}{cc}
-\left(\boldsymbol{\Phi}_{1}+\mathbf{\Psi}_{1}\right)+\lambda_{10} \boldsymbol{I} & \mathbf{0} \\
\mathbf{0} & t_{9}-\lambda_{10} \epsilon_{11}^{2}
\end{array}\right] \succeq \mathbf{0}, \quad \lambda_{10} \geq 0,} \\
& \boldsymbol{\Phi}_{1} \succeq \mathbf{0}, \quad \boldsymbol{\Psi}_{1} \succeq \mathbf{0}, \quad \operatorname{Tr}\left(\boldsymbol{\Phi}_{1}+\boldsymbol{\Psi}_{1}\right) \leq P_{1}, \\
& \boldsymbol{\Phi}_{2} \succeq \mathbf{0}, \quad \boldsymbol{\Psi}_{2} \succeq \mathbf{0}, \quad \operatorname{Tr}\left(\boldsymbol{\Phi}_{2}+\boldsymbol{\Psi}_{2}\right) \leq P_{2},
\end{aligned}
$$

where $t_{1}, t_{2}, \cdots, t_{10}$ are as defined in the optimization problem (38). The above problem can be easily solved using semidefinite programming techniques.

\section{RESUlTS AND DisCUSSIONS}

In this section, we present numerical results on the secrecy rate under perfect and imperfect CSI conditions. We assume that $M_{1}=M_{2}=2$. We have used the following channel gains as the estimates: $\boldsymbol{h}_{12}^{0}=[0.0838+0.5207 i, 0.2226-$ $0.2482 i], \boldsymbol{h}_{21}^{0}=[0.4407+0.6653 i, 0.5650-0.0015 i], \boldsymbol{z}_{1}^{0}=$ $[0.0765+0.0276 i,-0.0093+0.0062 i], \boldsymbol{z}_{2}^{0}=[-0.0449+$ $0.0314 i,-0.0396-0.0672 i]$. We assume that the magnitudes of the CSI errors in all the links are equal, i.e., $\epsilon_{11}=\epsilon_{12}=$ $\epsilon_{21}=\epsilon_{22}=\epsilon_{1}=\epsilon_{2}=\epsilon$. We also assume that $N_{0}=1$. In Fig. 2 and Fig. 3, we plot the $\left(R_{1}, R_{2}\right)$ region obtained

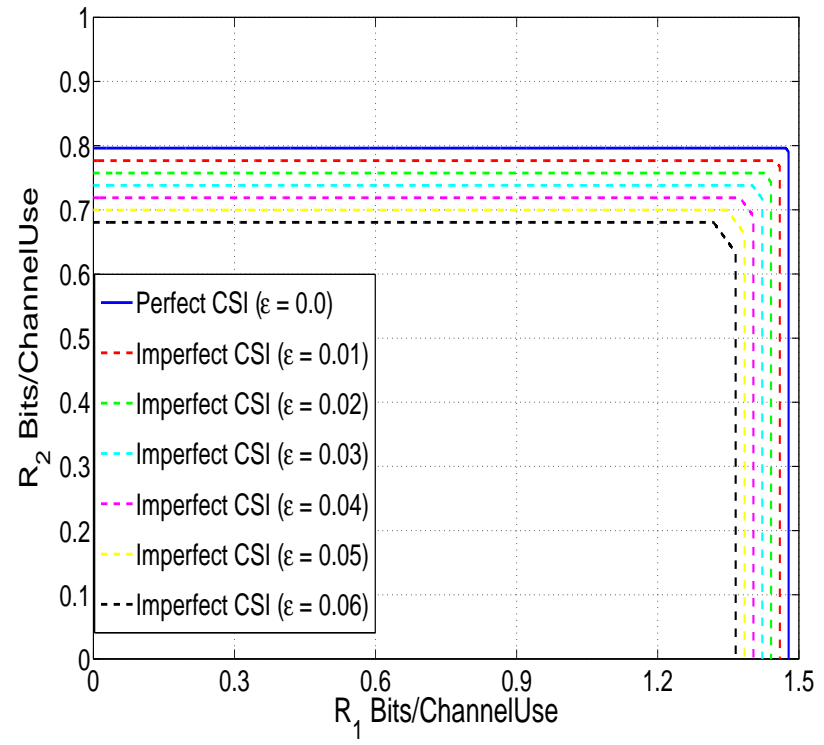

Fig. 2. Achievable $\left(R_{1}, R_{2}\right)$ region in full-duplex communication. $P_{1}=P_{2}=3 \mathrm{~dB}, M_{1}=M_{2}=2, N_{0}=1, \epsilon=$ $0.0,0.01,0.02,0.03,0.04,0.05,0.06$.

by maximizing the sum secrecy rate for various values of $\epsilon=0.0,0.01,0.02,0.03,0.04,0.05,0.06$. Results in Fig. 2 and Fig. 3 are generated for fixed powers $P_{1}=P_{2}=3 \mathrm{~dB}$ and $P_{1}=P_{2}=6 \mathrm{~dB}$, respectively. We observe that as the

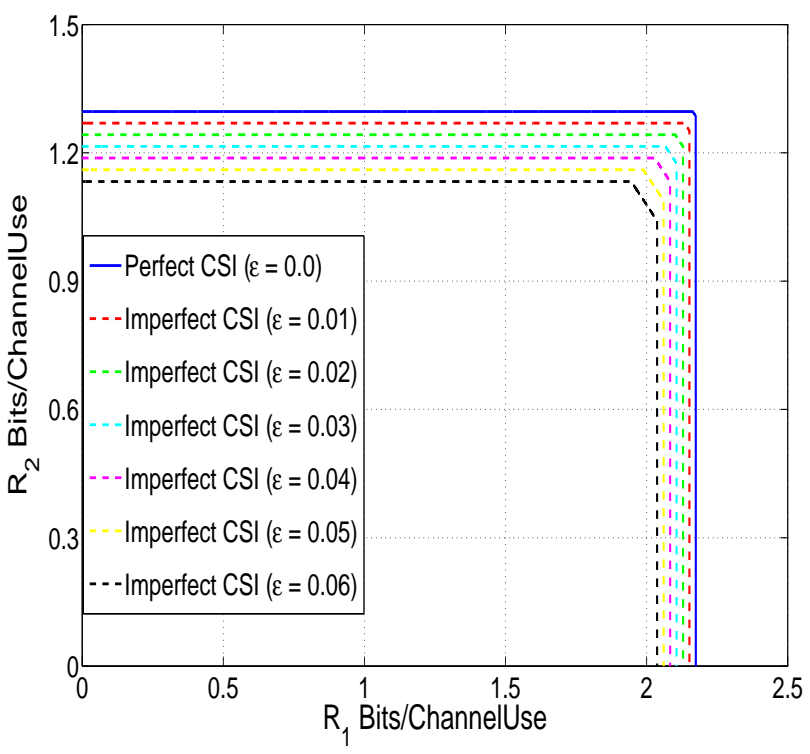

Fig. 3. Achievable $\left(R_{1}, R_{2}\right)$ region in full-duplex communication. $P_{1}=P_{2}=6 \mathrm{~dB}, M_{1}=M_{2}=2, N_{0}=1, \epsilon=$ $0.0,0.01,0.02,0.03,0.04,0.05,0.06$.

magnitude of the CSI error increases the corresponding sum secrecy rate decreases which results in the shrinking of the achievable rate region. Also, as the power is increased from $3 \mathrm{~dB}$ to $6 \mathrm{~dB}$, the achievable secrecy rate region increases.

\section{Conclusions}

We investigated the sum secrecy rate and the corresponding achievable secrecy rate region in MISO full-duplex wiretap channel when the CSI in all the links were assumed to be imperfect. We obtained the transmit covariance matrices associated with the message signals and the jamming signals which maximized the worst case sum secrecy rate. Numerical results illustrated the impact of imperfect CSI on the achievable secrecy rate region. We further note that transmit power optimization subject to outage constraint in a slow fading full-duplex MISO wiretap channel can be carried out using the approximations by conic optimization in [20] as future extension to this work.

\section{APPENDiX A}

In this appendix, we analyze the ranks of the solutions $\boldsymbol{\Phi}_{1}, \boldsymbol{\Psi}_{1}, \boldsymbol{\Phi}_{2}$, and $\boldsymbol{\Psi}_{2}$ which are obtained by solving the optimization problem (19) subject to the constraints in (20). We take the Lagrangian of the objective function $t$ subject to the constraints in (20) as follows [19]: 


$$
\begin{aligned}
& \ell\left(t, \boldsymbol{\Phi}_{1}, \boldsymbol{\Psi}_{1}, \boldsymbol{\Phi}_{2}, \boldsymbol{\Psi}_{2}, \lambda_{1}, \lambda_{2}, \boldsymbol{A}_{1}, \boldsymbol{B}_{1}, \boldsymbol{A}_{2}, \boldsymbol{B}_{2}, \mu, \nu_{1}, \nu_{2}\right)= \\
& \quad t+\lambda_{1}\left(\operatorname{Tr}\left(\boldsymbol{\Phi}_{1}+\boldsymbol{\Psi}_{1}\right)-P_{1}\right)+\lambda_{2}\left(\operatorname{Tr}\left(\boldsymbol{\Phi}_{2}+\boldsymbol{\Psi}_{2}\right)-P_{2}\right) \\
& \quad-\operatorname{Tr}\left(\boldsymbol{A}_{1} \boldsymbol{\Phi}_{1}\right)-\operatorname{Tr}\left(\boldsymbol{B}_{1} \boldsymbol{\Psi}_{1}\right)-\operatorname{Tr}\left(\boldsymbol{A}_{2} \boldsymbol{\Phi}_{2}\right)-\operatorname{Tr}\left(\boldsymbol{B}_{2} \boldsymbol{\Psi}_{2}\right) \\
& \quad+\mu\left(\left(\boldsymbol{z}_{1} \boldsymbol{\Phi}_{1} \boldsymbol{z}_{1}^{*}+\boldsymbol{z}_{2} \boldsymbol{\Phi}_{2} \boldsymbol{z}_{2}^{*}\right)-t\left(N_{0}+\boldsymbol{z}_{1} \boldsymbol{\Psi}_{1} \boldsymbol{z}_{1}^{*}+\boldsymbol{z}_{2} \boldsymbol{\Psi}_{2} \boldsymbol{z}_{2}^{*}\right)\right) \\
& \quad+\nu_{1}\left(\left(2^{R_{1}^{\prime}{ }^{k}}-1\right)\left(N_{0}+\boldsymbol{h}_{21} \boldsymbol{\Psi}_{1} \boldsymbol{h}_{21}^{*}\right)-\left(\boldsymbol{h}_{21} \boldsymbol{\Phi}_{1} \boldsymbol{h}_{21}^{*}\right)\right) \\
& \quad+\nu_{2}\left(\left(2^{R_{2}^{\prime l}}-1\right)\left(N_{0}+\boldsymbol{h}_{12} \boldsymbol{\Psi}_{2} \boldsymbol{h}_{12}^{*}\right)-\left(\boldsymbol{h}_{12} \boldsymbol{\Phi}_{2} \boldsymbol{h}_{12}^{*}\right)\right),
\end{aligned}
$$

where $\lambda_{1} \geq 0, \lambda_{2} \geq 0, \boldsymbol{A}_{1} \succeq \mathbf{0}, \boldsymbol{B}_{1} \succeq \mathbf{0}, \boldsymbol{A}_{2} \succeq \mathbf{0}, \boldsymbol{B}_{2} \succeq \mathbf{0}$, $\mu \geq 0, \nu_{1} \geq 0$, and $\nu_{2} \geq 0$ are the Lagrangian multipliers. The KKT conditions of (66) are as follows:

(a1) all the constraints in 20,

(a2) $\lambda_{1}\left(\operatorname{Tr}\left(\boldsymbol{\Phi}_{1}+\boldsymbol{\Psi}_{1}\right)-P_{1}\right)=0$

(a3) $\lambda_{2}\left(\operatorname{Tr}\left(\boldsymbol{\Phi}_{2}+\boldsymbol{\Psi}_{2}\right)-P_{2}\right)=0$,

(a4) $\operatorname{Tr}\left(\boldsymbol{A}_{1} \boldsymbol{\Phi}_{1}\right)=0$. Since $\boldsymbol{A}_{1} \succeq \mathbf{0}$ and $\boldsymbol{\Phi}_{1} \succeq \mathbf{0} \Longrightarrow$ $A_{1} \Phi_{1}=0$

(a5) $\operatorname{Tr}\left(\boldsymbol{B}_{1} \boldsymbol{\Psi}_{1}\right)=0$. Since $\boldsymbol{B}_{1} \succeq \mathbf{0}$ and $\boldsymbol{\Psi}_{1} \succeq \mathbf{0} \Longrightarrow$ $\boldsymbol{B}_{1} \boldsymbol{\Psi}_{1}=\mathbf{0}$

(a6) $\operatorname{Tr}\left(\boldsymbol{A}_{2} \boldsymbol{\Phi}_{2}\right)=0$. Since $\boldsymbol{A}_{2} \succeq \mathbf{0}$ and $\boldsymbol{\Phi}_{2} \succeq \mathbf{0} \Longrightarrow$ $\boldsymbol{A}_{2} \boldsymbol{\Phi}_{2}=\mathbf{0}$,

(a7) $\operatorname{Tr}\left(\boldsymbol{B}_{2} \boldsymbol{\Psi}_{2}\right)=0$. Since $\boldsymbol{B}_{2} \succeq \mathbf{0}$ and $\boldsymbol{\Psi}_{2} \succeq \mathbf{0} \Longrightarrow$ $\boldsymbol{B}_{2} \boldsymbol{\Psi}_{2}=\mathbf{0}$,

(a8) $\mu\left(\left(\boldsymbol{z}_{1} \boldsymbol{\Phi}_{1} \boldsymbol{z}_{1}^{*}+\boldsymbol{z}_{2} \boldsymbol{\Phi}_{2} \boldsymbol{z}_{2}^{*}\right)-t\left(N_{0}+\boldsymbol{z}_{1} \boldsymbol{\Psi}_{1} \boldsymbol{z}_{1}^{*}+\boldsymbol{z}_{2} \boldsymbol{\Psi}_{2} \boldsymbol{z}_{2}^{*}\right)\right)=0$, (a9) $\nu_{1}\left(\left(2^{R_{1}^{\prime k}}-1\right)\left(N_{0}+\boldsymbol{h}_{21} \boldsymbol{\Psi}_{1} \boldsymbol{h}_{21}^{*}\right)-\left(\boldsymbol{h}_{21} \boldsymbol{\Phi}_{1} \boldsymbol{h}_{21}^{*}\right)\right)=0$, (a10) $\nu_{2}\left(\left(2^{R_{2}^{\prime} l}-1\right)\left(N_{0}+\boldsymbol{h}_{12} \boldsymbol{\Psi}_{2} \boldsymbol{h}_{12}^{*}\right)-\left(\boldsymbol{h}_{12} \boldsymbol{\Phi}_{2} \boldsymbol{h}_{12}^{*}\right)\right)=0$,

(a11) $\frac{\partial \ell}{\partial t}=0 \Longrightarrow \mu\left(N_{0}+\boldsymbol{z}_{1} \boldsymbol{\Psi}_{1} \boldsymbol{z}_{1}^{*}+\boldsymbol{z}_{2} \boldsymbol{\Psi}_{2} \boldsymbol{z}_{2}^{*}\right)=1$. This implies that $\mu>0$,

(a12) $\frac{\partial \ell}{\partial \boldsymbol{\Phi}_{1}}=\mathbf{0} \Longrightarrow \boldsymbol{A}_{1}=\lambda_{1} \boldsymbol{I}+\mu \boldsymbol{z}_{1}^{*} \boldsymbol{z}_{1}-\nu_{1} \boldsymbol{h}_{21}^{*} \boldsymbol{h}_{21} \succeq \mathbf{0}$,

(a13) $\frac{\partial \ell}{\partial \boldsymbol{\Psi}_{1}}=\mathbf{0} \Longrightarrow \boldsymbol{B}_{1}=\lambda_{1} \boldsymbol{I}-\mu t \boldsymbol{z}_{1}^{*} \boldsymbol{z}_{1}+\nu_{1}\left(2^{R_{1}^{\prime k}}-\right.$ 1) $\boldsymbol{h}_{21}^{*} \boldsymbol{h}_{21} \succeq \mathbf{0}$,

(a14) $\frac{\partial \ell}{\partial \boldsymbol{\Phi}_{2}}=\mathbf{0} \Longrightarrow \boldsymbol{A}_{2}=\lambda_{2} \boldsymbol{I}+\mu \boldsymbol{z}_{2}^{*} \boldsymbol{z}_{2}-\nu_{2} \boldsymbol{h}_{12}^{*} \boldsymbol{h}_{12} \succeq \mathbf{0}$,

(a15) $\frac{\partial \ell}{\partial \boldsymbol{\Psi}_{2}}=\mathbf{0} \Longrightarrow \boldsymbol{B}_{2}=\lambda_{2} \boldsymbol{I}-\mu t \boldsymbol{z}_{2}^{*} \boldsymbol{z}_{2}+\nu_{2}\left(2^{R_{2}^{\prime}}-\right.$ 1) $\boldsymbol{h}_{12}^{*} \boldsymbol{h}_{12} \succeq \mathbf{0}$.

We first consider the scenario when $\lambda_{1}>0$. The KKT condition (a12) implies that

$$
\boldsymbol{A}_{1}+\nu_{1} \boldsymbol{h}_{21}^{*} \boldsymbol{h}_{21}=\lambda_{1} \boldsymbol{I}+\mu \boldsymbol{z}_{1}^{*} \boldsymbol{z}_{1} \succ \mathbf{0} .
$$

The above expression implies that $\operatorname{rank}\left(\boldsymbol{A}_{1}\right) \geq M_{1}$ $\operatorname{rank}\left(\nu_{1} \boldsymbol{h}_{21}^{*} \boldsymbol{h}_{21}\right)$. Since $\operatorname{rank}\left(\nu_{1} \boldsymbol{h}_{21}^{*} \boldsymbol{h}_{21}\right) \leq 1$, this further implies that $\operatorname{rank}\left(\boldsymbol{A}_{1}\right) \geq M_{1}-1$. Assuming $\boldsymbol{\Phi}_{1} \neq \mathbf{0}$, the KKT condition (a4) implies that $\operatorname{rank}\left(\boldsymbol{A}_{1}\right)=M_{1}-1$, and the expression 67) implies that $\nu_{1}>0$. This means that $\operatorname{rank}\left(\boldsymbol{\Phi}_{1}\right)=1$. With $\lambda_{1}>0$, and $\nu_{1}>0$, we rewrite the KKT condition (a13) in the following form:

$$
\boldsymbol{B}_{1}+\mu t \boldsymbol{z}_{1}^{*} \boldsymbol{z}_{1}=\lambda_{1} \boldsymbol{I}+\nu_{1}\left(2^{R_{1}^{\prime k}}-1\right) \boldsymbol{h}_{21}^{*} \boldsymbol{h}_{21} \succ \mathbf{0} .
$$

If $t>0$, the above expression implies that $\operatorname{rank}\left(\boldsymbol{B}_{1}\right) \geq$ $M_{1}-\operatorname{rank}\left(\mu t z_{1}^{*} z_{1}\right)=M_{1}-1$. The KKT condition (a5) implies that $\operatorname{rank}\left(\boldsymbol{B}_{1}\right)=M_{1}-1$, and $\operatorname{rank}\left(\boldsymbol{\Psi}_{1}\right)=1$ (assuming $\boldsymbol{\Psi}_{1} \neq \mathbf{0}$ ). Now, if $t=0$, the KKT condition (a8) implies that $\boldsymbol{z}_{1} \boldsymbol{\Phi}_{1} \boldsymbol{z}_{1}^{*}+\boldsymbol{z}_{2} \boldsymbol{\Phi}_{2} \boldsymbol{z}_{2}^{*}=0$, i.e., the received signal power at the eavesdropper will be zero. The expression (68), and the KKT condition (a5) further imply that $\boldsymbol{\Psi}_{1}=\mathbf{0}$. Also, when $\lambda_{1}>0$, the KKT condition (a2) implies that $\operatorname{Tr}\left(\boldsymbol{\Phi}_{1}+\boldsymbol{\Psi}_{1}\right)=P_{1}$, i.e., the entire power $P_{1}$ is used for the transmission. Similar rank analysis holds for $\boldsymbol{\Phi}_{2}$ and $\boldsymbol{\Psi}_{2}$ when $\lambda_{2}>0$.

We now consider the scenario when $\lambda_{1}=0$. Assuming $z_{1}$ and $\boldsymbol{h}_{21}$ are not collinear, the KKT condition (a12) will be satisfied only when $\nu_{1}=0$. With this, the expression 677 implies that $\boldsymbol{A}_{1}=\mu \boldsymbol{z}_{1}^{*} \boldsymbol{z}_{1}$ and $\operatorname{rank}\left(\boldsymbol{A}_{1}\right)=\operatorname{rank}\left(\mu \boldsymbol{z}_{1}^{*} \boldsymbol{z}_{1}\right)=$ 1. The KKT condition (a4) further implies that the eigen vectors corresponding to the non-zero eigen values of $\boldsymbol{\Phi}_{1}$ lie in the orthogonal complement subspace of $\boldsymbol{z}_{1}^{*}$, and $\operatorname{rank}\left(\boldsymbol{\Phi}_{1}\right) \leq M_{1}-1$. Further, with $\lambda_{1}=0$ and $\nu_{1}=0$, the KKT condition (a13) will be satisfied only when $t=0$ i.e., $\boldsymbol{z}_{1} \boldsymbol{\Phi}_{1} \boldsymbol{z}_{1}^{*}+\boldsymbol{z}_{2} \boldsymbol{\Phi}_{2} \boldsymbol{z}_{2}^{*}=0$. The above analysis implies that there exist a rank-1 optimum $\boldsymbol{\Phi}_{1}$. Similar rank analysis holds for $\boldsymbol{\Phi}_{2}$ and $\boldsymbol{\Psi}_{2}$ when $\lambda_{2}=0$.

\section{REFERENCES}

[1] A. Wyner, "The wire-tap channel," Bell. Syst Tech. J, vol. 54, no. 8, pp. 1355-1387, Jan. 1975.

[2] I. Csiszar and J. Korner, "Broadcast channels with confidential messages," IEEE Trans. Inform. Theory, pp. 339-348, May 1978.

[3] S. K. Leung-Yan-Cheong and M. E. Hellman, "The Gaussian wire-tap channel," IEEE Trans. Inform. Theory, pp. 451-456, Jul. 1978

[4] Y. Liang, H. V. Poor, and S. Shamai (Shitz), "Information theoretic security," Foundations and Trends in Communications and Information Theory, NOW Publishers, vol. 5, no. 4-5, 2009.

[5] S. Shafiee and S. Ulukus, "Achievable rates in Gaussian MISO channels with secrecy constraint," Proc. IEEE ISIT'2007, June 2007.

[6] F. Oggier and B. Hassibi, "The secrecy capacity of the MIMO wiretap channel," Proc. IEEE ISIT'2008, July 2008.

[7] A. Khisti and G. Wornell, "Secure transmission with multiple antennasII: The MIMOME wiretap channel," IEEE Trans. Inform. Theory, vol. 56, no. 7, pp. 3088-3104, Jul. 2010.

[8] A. Sabharwal, P. Schniter, D. Guo, D. W. Bliss, S. Rangarajan, and R. Wichman, "In-band full-duplex wireless: challenges and opportuni-

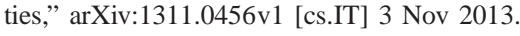

[9] M. Duarte and A. Sabharwal, "Full-duplex wireless communications using off-the-shelf radios: feasibility and first results," Conference Record of the Forty Fourth Asilomar Conference on Signals, Systems and Computers (ASILOMAR), pp. 1558-1562, Nov. 2010.

[10] T. Riihonen, S. Werner, and R. Wichman, "Hybrid full-duplex/halfduplex relaying with transmit power adaptation," IEEE Trans. Wireless Commun., vol. 10, no. 9, pp. 3074-3085, Sep. 2011.

[11] T. Riihonen, S. Werner, and R. Wichman, "Mitigation of loopback selfinterference in full-duplex MIMO relays," IEEE Trans. Signal Proc., vol. 59, no. 12, pp. 5983-5993, Dec. 2011.

[12] A. Thangaraj, R. K. Ganti and S. Bhashyam, "Self-interference cancellation models for full-duplex wireless communications," Proc. SPCOM'2012, July 2012.

[13] E. Tekin and A. Yener, "The general Gaussian multiple-access and two-way wiretap channels: achievable rates and cooperative jamming," IEEE Trans. Inform. Theory, vol. 54, no. 6, pp. 2735-2751, Jun. 2008.

[14] E. Tekin and A. Yener, "Correction to: "The Gaussian multiple-access wire-tap channel" and "The general Gaussian multiple-access and twoway wiretap channels: achievable rates and cooperative jamming"," IEEE Trans. Inform. Theory, vol. 56, no. 9, pp. 4762-4763, Sep. 2010. 
[15] A. E. Gamal, O. O. Koyluoglu, M. Youssef, and H. E. Gamal, "Achievable secrecy rate regions for the two-way wiretap channel," IEEE Trans. Inform. Theory, vol. 59, no. 12, pp. 8099-8114, Dec. 2013.

[16] Q. Li and W. K. Ma, "Optimal and robust transmit designs for MISO channel secrecy by semidefinite programming," IEEE Trans. Signal Process., vol. 59, no. 8, pp. 3799-3812, Aug. 2011.

[17] J. Huang and A. L. Swindlehurst, "Robust secure transmission in MISO channels based on worst-case optimization," IEEE Trans. Signal Process., vol. 60, no. 4, pp. 1696-1707, Apr. 2012.

[18] Q. Li and W. K. Ma, "Spatially selective artificial-noise aided transmit optimization for MISO multi-eves secrecy rate maximization," IEEE Trans. Signal Process., vol. 61, no. 10, pp. 2704-2717, Mar. 2013.

[19] S. Boyd and L. Vandenberghe, Convex optimization, Cambridge Univ. Press, 2004.

[20] K-Y. Wang, A. M-C. So, T-H. Chang, W-K. Ma, and C-Y. Chi, "Outage constrained robust transmit optimization for multiuser MISO downlinks: tractable approximations by conic optimization," IEEE Trans. Signal Process., vol. 62, no. 21, pp. 5690-5705, Nov. 2014. 